Original Article

\title{
Effect of maximum voluntary isometric contraction of antagonist muscles in rate of torque development of agonist muscles
}

\author{
ELEFTHERIA BAKALOUDI ${ }^{1}$, CHRYSTALLA PANTELI $^{1}$, ANTHI XENOFONTOS ${ }^{1}$, ELENI BASSA ${ }^{1}$, \\ CHRISTOS KOTZAMANIDIS ${ }^{1}$ \\ ${ }^{1}$ Laboratory of Coaching and Sport Performance, Department of Physical Education and Sport Science, \\ Aristotle University of Thessaloniki, Thessaloniki, GREECE
}

Published online: September 21, 2012

(Accepted for publication July 10 2012)

DOI:10.7752/jpes.2012.03040; http://dx.doi.org/10.7752/jpes.2012.03040

\begin{abstract}
The purpose of this study was to investigate the effect of antagonist maximum voluntary isometric contraction on the rate of torque development of agonist muscles in the ankle joint. Fifteen healthy women $(22.03 \pm 1.78 \mathrm{y})$ were evaluated. An isokinetic dynamometer CYBEX Norm was used for the evaluation of torque. Initially the subjects performed 5 maximal plantar flexion isometric contractions of 2 s duration (phase 1 ) and then 5 maximal dorsiflexion isometric contractions for $2 \mathrm{~s}$ and immediately after it, a maximal plantar flexion for $2 \mathrm{~s}$ (phase 2, reversal). One minute rest interval was given between all trials and the best trial of phase 1 and phase 2 were compared for the following parameters: Maximal Voluntary Isometric Contraction (MVIC), maximal Rate of Torque Development (RTD) and RTD in the period 0-30, 0-50, 0-100 and 0-200 ms after the torque onset. Statistical analysis showed that RTD, and RTD at $0-100 \mathrm{~ms}$ was higher in phase 2 compared to phase 1 $(p<0.001)$, whereas no effects were observed in MVIC $(p>0.05)$. In conclusion, it was found that reversal of antagonists affects positively the rate of torque development in plantar flexors especially during the first $100 \mathrm{~ms}$ of torque onset.

Keywords: Maximum voluntary contraction, RFD, reversal, plantar flexors
\end{abstract}

\section{Introduction}

The Rate of Torque Development (RTD) shows the change of torque in time, from the onset of torque to the time of appearance the maximum isometric torque. It is influenced both by neuronal and muscle factors as well (Andersen \& Aagaard, 2006). However, it has been proposed (Grabiner, 1994) that RTD of agonist muscles is enhanced when a maximal contraction of the antagonist muscles is preceded, based on the phenomenon of the "reversal of antagonist muscles" (REV).

The idea of REV was introduced by Kabat (1950) and it was based on Sherrington's concept (1947) that elementary reflexes interact to trigger a more complex process to coordinate agonist and antagonist action. For this reason, REV was based on Golgi tendon organs (GTOs) function, which as previously has been reported (Carew, 1982), when a muscle is contracted inhibits the function of agonist muscle and facilitates the antagonist, through Ib afferent fibers.

The maximal inhibition from GTOs occurs between 1s after contraction (Moore \& Kukulka, 1991; Garceau, Fauth, Hanson, Hsu, Yoon, Szalkowski, Lutsch \& Ebben, 2010). That's why the duration of the antagonist contraction acting for the potentiation of agonist muscle in REV occurrence should last more than $1 \mathrm{~s}$ (Kamimura \& Takenaka, 2007). The exact nature of this mechanism has not experimentally been identified thaw several neuronal or muscular mechanisms have been proposed (Roy, Sylvestre, Katch F.I., Katch V.L. \& Lagassé, 1990).

Regarding the effect of REV on muscle performance two cases have been studied: the peak torque and the RTD. Initially the REV effect was tested in hemiparetic subjects were peak torque enhancement was observed (Bohannon, 1985). However, it was not the case for healthy people were conflicting results have been reported because in other cases enhancement was reported (Roy et al., 1990; Kamimura, Yoshioka, Ito, \& Kusakabe, 2009; Kamimura et al., 2007) while in others not (Bohannon, Gibson \& Larkin, 1986; Gabriel, Basford, \& An., 2001; Grabiner, 1994).

The above mentioned studies did not measure the effect of the antagonist reversal on the phases of RTD so it is not known yet which part is affected more. So the primary aim of this study was to re-examine the effect of maximum voluntary isometric contraction of antagonist muscles on the rate of torque development of 
agonist muscles in the ankle joint. Secondary aim was to reveal in which time phases of RTD possible changes were observed.

\section{Material and method}

\section{Participants}

The sample consisted of 15 healthy women, who were students of the Department of Physical Education and Sport Science. They were aged from 19 to 26 years old (mean \pm SD: $22.03 \pm 1.78$ years), body mass from 59 to $70.5 \mathrm{~kg}$ (mean \pm SD: $62.89 \pm 5.01 \mathrm{~kg}$ ) and height from 153 to $174 \mathrm{~cm}$ (mean \pm SD: $165.27 \pm$ $6.03 \mathrm{~cm}$ ). All participants were familiar with the testing protocol and they gave their written consent, having been informed of the process. The experimental process was complied with the Ethics standards provided by the Aristotle University of Thessaloniki.

\section{Instrumentation}

Dynamometry: The isokinetic dynamometer CYBEX Norm (Lumex Corporation, Ronkohoma, NY), according to manufacturing instructions regarding the alignment of the specific platform that has the dynamometer for the measurement of the ankle joint.

\section{Experimental Protocol}

The experimental protocol was consisted of two stages. Participants in the first stage performed 3 maximum isometric contractions of the plantar flexors for $2 \mathrm{~s}, 1$ minute rest period and 3 maximum isometric contractions of the dorsiflexors for $2 \mathrm{~s}$ with 1 minute rest period between them. They rested for 10 minutes off the machine and they performed the second stage of the protocol.

Participants in second stage performed 5 maximal plantar flexion isometric contractions for $2 \mathrm{~s}$ and 1 minute rest (phase1). Then they performed a maximal dorsiflexion isometric contraction for $2 \mathrm{~s}$ and immediately after it, a maximal plantar flexion for $2 \mathrm{~s}$ (phase2). The whole cycle was performed five times with 1 min. interval between cycles.

\section{Testing}

Participants sat on the chair of CYBEX with their back almost lying and placed their right foot on a platform, with their knee stretched $\left(180^{\circ}\right)$ and their ankle joint in anatomical zero $\left(90^{\circ}\right)$. The straps were placed in order to immobilize their foot on the platform and the femur, pelvis and torso to the seat of the machine. Their hands were on their chest crosswise, so as not to assist during the contraction.

During the experimental process the execution instructions were given for "as fast as possible" (Sahaly, Vandewalle \& Driss, 2001)

\section{Analysis of data}

For the analysis of the torque curve was defined as starting point of the contraction (onset) that moment when resting line of the torque was increased by $2.5 \%$ to the maximum torque value, which was recorded in this effort (Aagaard, Simonsen, Andersen, Magnusson, \& Dyhre-Poulsen, 2002).

The method of calculating the rate of torque development of voluntary torque that was followed was the following: the torque curve for the unit of time, from the beginning of the torque line (point 0 ) to $30,50,100$, and $200 \mathrm{~ms}$.

\section{Statistical Analysis}

The program Statistica was used for the statistical analysis. As dependent variables were defined: the values of maximum torque, maximum RTD, the time of appearance of maximum torque, the time of appearance of maximum RTD, whereas the independent variables were: the time of contraction, the type of contraction (isometric, only maximum, or with reversal). In the analysis of data was used the model of analysis of variance: ANOVA for repeated measurements. For the post-hoc analysis was used the Scheffe test. The significance level was set at $\mathrm{p}<0.05$.

\section{Results}

From the statistical analysis a statistically significant effect of the execution mode on the maximum torque was observed $\mathrm{F}_{(1,14)}=268.55, \mathrm{p}<0.01$ between maximal isometric contractions and reversals of antagonists (as shown in Figure I). In particular, at the fourth attempt the maximum torque during maximum contraction is appeared to be statistically significantly higher $\mathrm{p}<0.01$ compared to the value of the maximum torque at the reversal. It was not observed any effect of the trial in maximum torque $(\mathrm{p}>0.05)$ or interaction between execution mode and trial $(\mathrm{p}>0.05)$. 


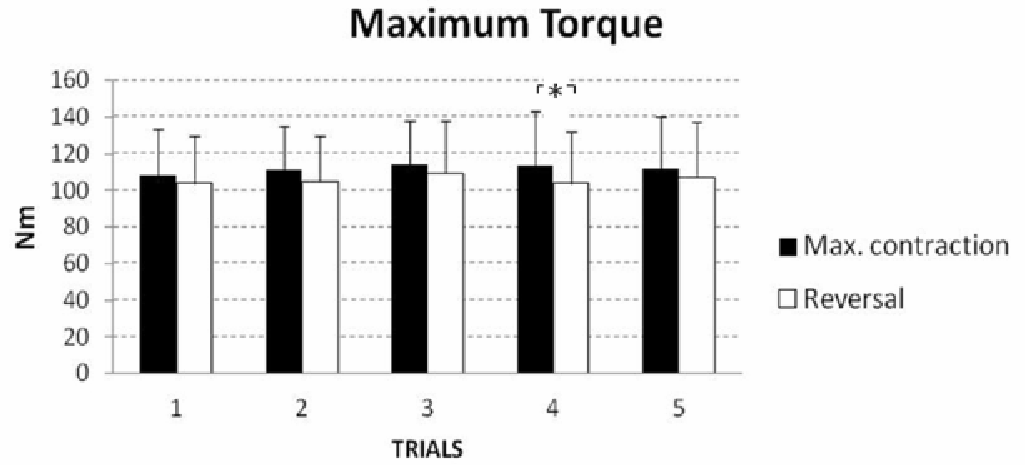

Figure I: The maximum torque at the maximal voluntary isometric contraction (black bar) and at the reversal of antagonists (white bar)

Statistical analysis showed no effect of the execution mode in the time of appearance of maximum torque, ( $>0.05$, as shown in Figure II). Moreover, there was no effect of the trial at the time of appearance of maximum torque $(\mathrm{p}>0.05)$ or interaction between the execution mode and the trial $(\mathrm{p}>0.05)$.

\section{Time of appearance of maximum torque}

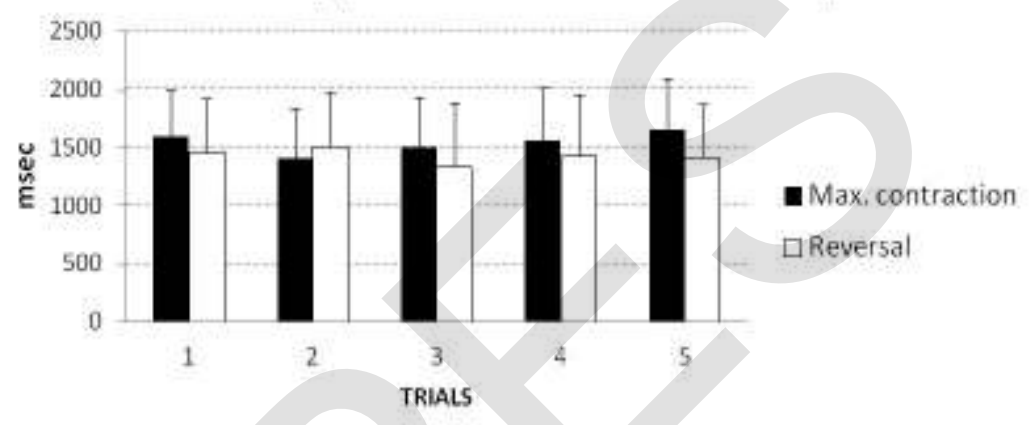

Figure II: The time of appearance of maximum torque at the maximal voluntary isometric contraction (black bar) and at the reversal of antagonists (white bar)

From the statistical analysis was an observed effect of the execution mode on the rate of torque development $\mathrm{F}$ $(1,14)=12.485, \mathrm{p}<0.001$ (as shown in Figure III). There was no effect of the trial in the rate of torque development $(\mathrm{p}>0.05)$ or interaction between the execution mode and the trial $(\mathrm{p}>0.05)$. The maximum rate of torque development appears to be statistically significantly higher, $\mathrm{p}<0.001$ at the reversal compared to the value of the maximum voluntary isometric contractions during all the trials except the fifth trial, where there are no significant differences $(\mathrm{p}>0.05)$.

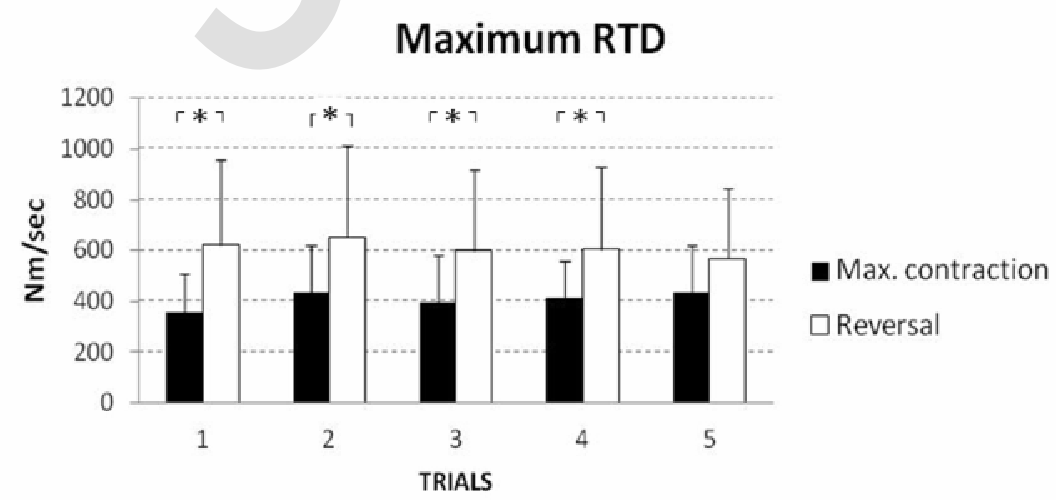

Figure III: The maximum rate of torque development at the maximal voluntary isometric contraction (black bar) and at the reversal of antagonists (white bar)

From the statistical analysis was observed effect of the execution mode at the time of appearance of rate of torque development, $\mathrm{F}_{(1,14)}=9,0000,(\mathrm{p}<0.01)$ (as shown in Figure IV). There was no effect of the trial at the time of appearance of rate of torque development $(\mathrm{p}>0.05)$ or interaction between the execution mode and the trial $(\mathrm{p}>0.05)$. 


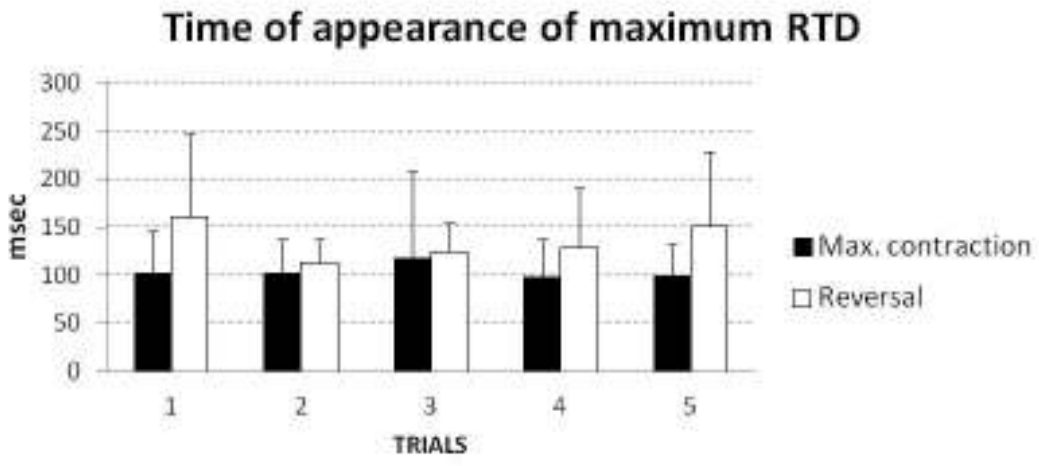

Figure IV: Time of appearance of maximum rate of torque development at the maximal voluntary isometric contraction (black bar) and at the reversal of antagonists (white bar)

From the statistical analysis was an observed effect of the execution mode on the torque development on the first $30 \mathrm{~ms} \mathrm{~F}_{(1,14)}=16,510,(\mathrm{p}<0.01$, as shown in Figure V). The torque at the first $30 \mathrm{~ms}$ appears to be statistically significantly higher, $\mathrm{p}<0.01$ at the maximum voluntary isometric contractions compared to reversal. However there was no effect of the trial to the development of the torque at the first $30 \mathrm{~ms}$ ( $>>0.05)$, or interaction between the execution mode and the trial $(\mathrm{p}>0.05)$.

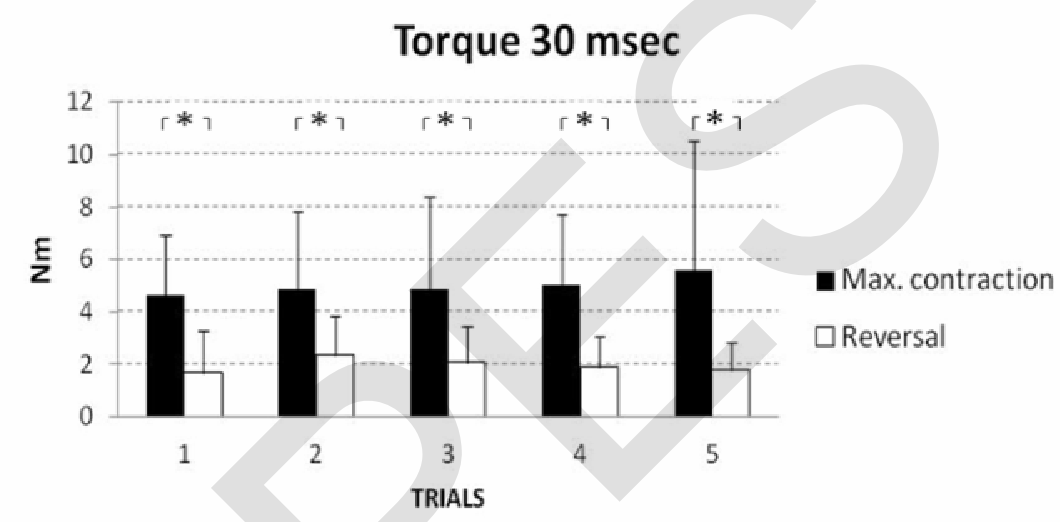

Figure V: The development of the torque development at the first $30 \mathrm{~ms}$ at the maximal voluntary isometric contraction (black bar) and at the reversal of antagonists (white bar)

From the statistical analysis was an observed effect of the execution mode on the torque development on the first $50 \mathrm{~ms} \mathrm{~F}_{(1,14)}=13.892,(\mathrm{p}<0.01$, as shown in Figure VI). In particular, at the first, fourth and fifth attempt the torque appears to be statistically significantly higher, $\mathrm{p}<0.01$ compared with the development of torque at the reversal. It was not observed any effect of the trial in the development of the torque at the first $50 \mathrm{~ms}$ $(\mathrm{p}>0.05)$ or interaction between execution mode and trial $(\mathrm{p}>0.05)$.

\section{Torque 50 msec}

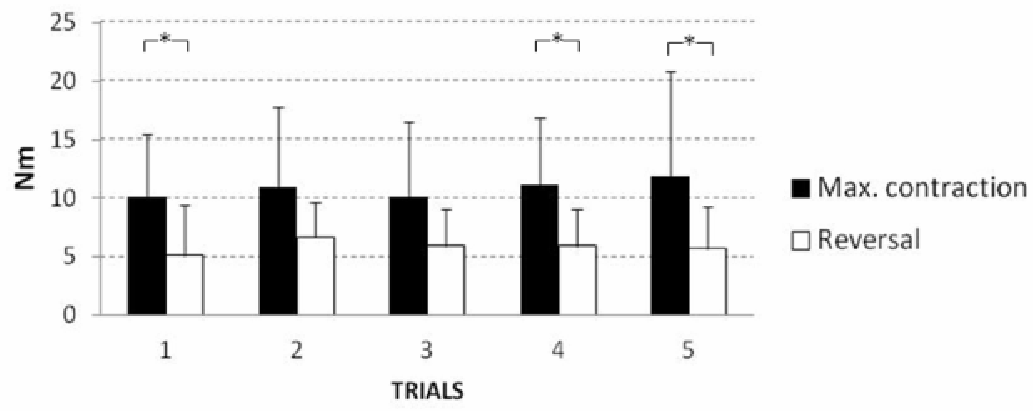

Figure VI: The development of the torque development at the first $50 \mathrm{~ms}$ at the maximal voluntary isometric contraction (black bar) and at the reversal of antagonists (white bar) 
Statistical analysis showed no effect neither of the execution mode nor of the trial at the torque development at the first $100 \mathrm{~ms}(\mathrm{p}>0.051$, as shown in Figure VII). Finally it was not observed any interaction between execution mode and trial $(\mathrm{p}>0.05)$.

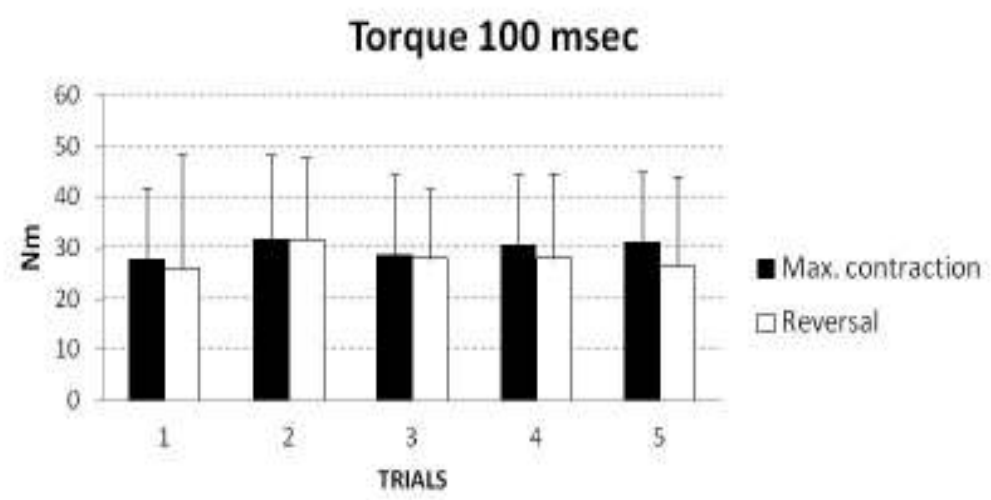

Figure VII: The development of the torque development at the first $100 \mathrm{~ms}$ at the maximal voluntary isometric contraction (black bar) and at the reversal of antagonists (white bar)

Statistical analysis showed no effect of the execution mode in the torque development at the first $200 \mathrm{~ms}$ of maximum torque, $(p>0.05$, as shown in Figure VIII). However, the effect of the trial was found in the torque development at the first $200 \mathrm{~ms}$, where $\mathrm{F}_{(4,56)}=435,8,(\mathrm{p}<0.05)$. Finally it was not observed any statistically significant interaction between execution mode and trial $(\mathrm{p}>0.05)$.

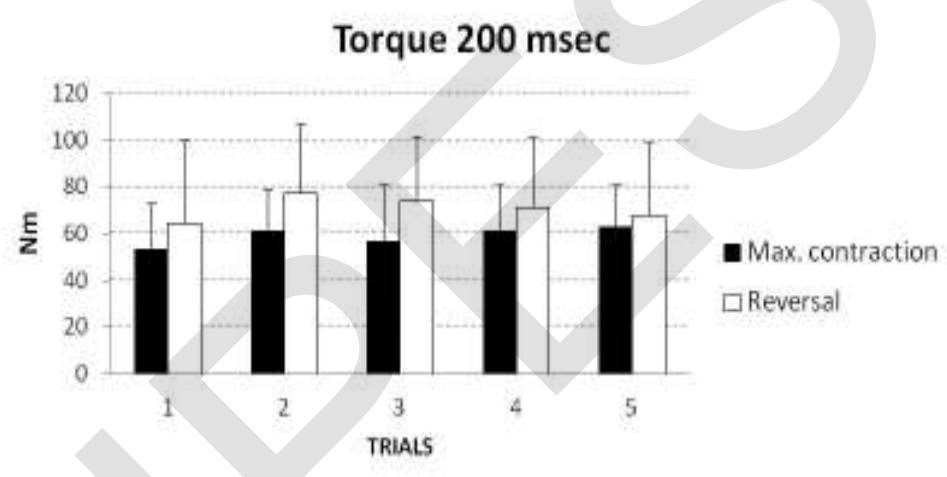

Figure VIII: The development of the torque development at the first $200 \mathrm{~ms}$ at the maximal voluntary isometric contraction (black bar) and at the reversal of antagonists (white bar)

From the statistical analysis was an observed effect of the execution mode of the rate of torque development at the first $30 \mathrm{~ms}, \mathrm{~F}_{(1,14)}=16,510,(\mathrm{p}<0.005)$. In particular, the rate of torque development appears to be statistically significantly higher at the maximal isometric contractions, $(p<0.005)$ compared with the rate of torque development at the reversal (as shown in Figure IX). There was no statistically significant effect of the trial on the development of the rate of torque development in the first $30 \mathrm{~ms}(\mathrm{p}>0.05)$, or interaction between the execution mode and the trial $(\mathrm{p}>0.05)$.

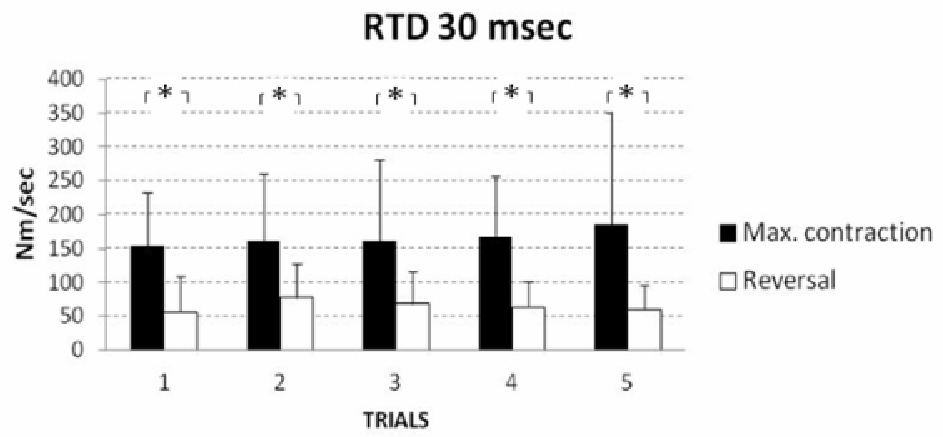

Figure IX: The rate of torque development at the first $30 \mathrm{~ms}$ at the maximal voluntary isometric contraction (black bar) and at the reversal of antagonists (white bar) 
From the statistical analysis was observed effect of the execution mode of the rate of torque development at the first $50 \mathrm{~ms}, \mathrm{~F}_{(1,14)}=13.892, \mathrm{p}<0.005$ (as shown in Figure $\mathrm{X}$ ). It was not observed any effect of the trial in the rate of torque development at the first $50 \mathrm{~ms} \mathrm{p}<0.005$ or any interaction between execution mode and trial $(\mathrm{p}>0.05)$. The rate of torque development at the first $50 \mathrm{~ms}$ appears to be statistically significantly higher, $\mathrm{p}<0.005$ at the maximal isometric contractions at the first, fourth and fifth trial compared with the rate of torque development at the reversal.

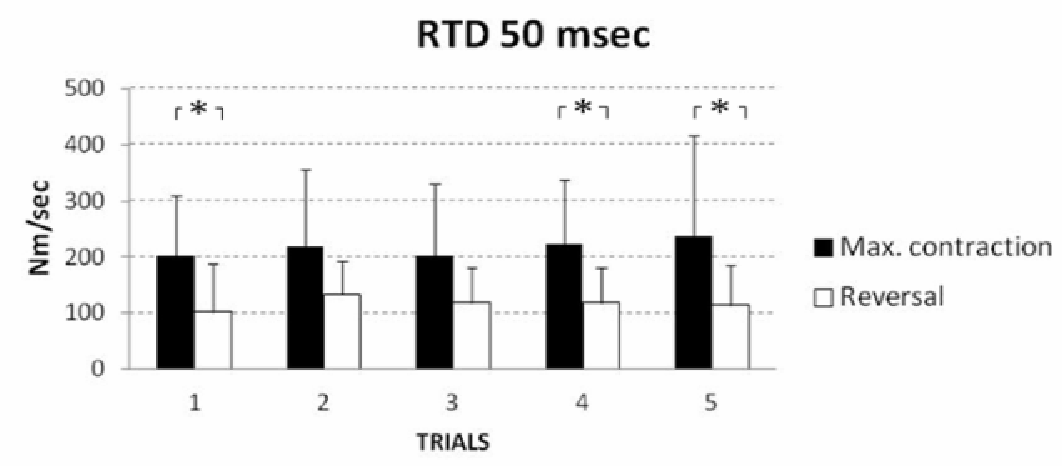

Figure X: The rate of torque development at the first $50 \mathrm{~ms}$ at the maximal voluntary isometric contraction (black bar) and at the reversal of antagonists (white bar)

Statistical analysis showed no effect neither of the execution mode nor of the trial at the rate of torque development at the first $100 \mathrm{~ms}(\mathrm{p}>0.05$, as shown in Figure XI). Moreover it was not observed any interaction between execution mode and trial $(\mathrm{p}>0.05)$.

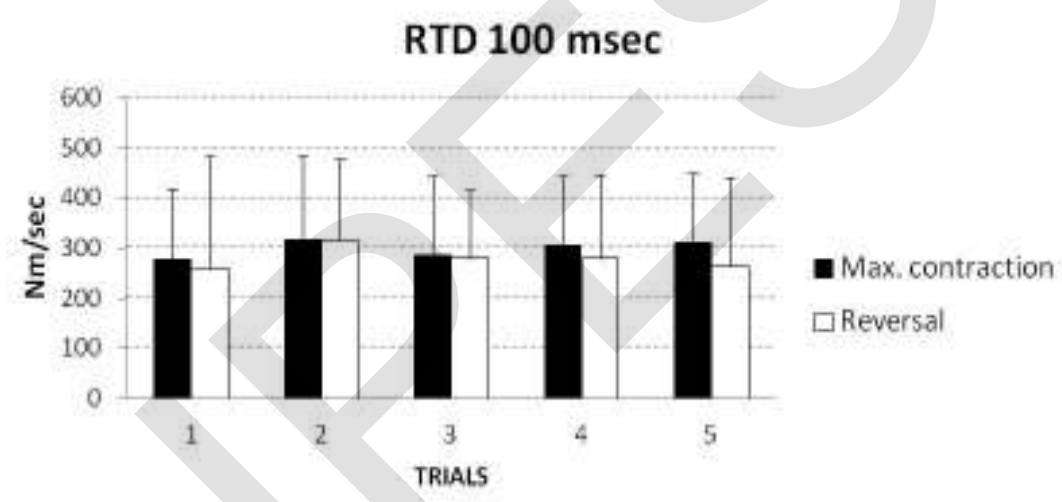

Figure XI: The rate of torque development at the first $100 \mathrm{~ms}$ at the maximal voluntary isometric contraction (black bar) and at the reversal of antagonists (white bar)

Statistical analysis showed no effect of the execution mode at the rate of torque development at the first $200 \mathrm{~ms}$ $(p>0.05)$. However it was observed statistically significant effect of the trial to the rate of torque development at the first $200 \mathrm{~ms}\left(\mathrm{~F}_{(1,14)}=151.42, \mathrm{p}<0.05\right.$, as shown in Figure XII), whereas it was not observed any interaction of the execution mode and the trial $(\mathrm{p}>0.05)$.

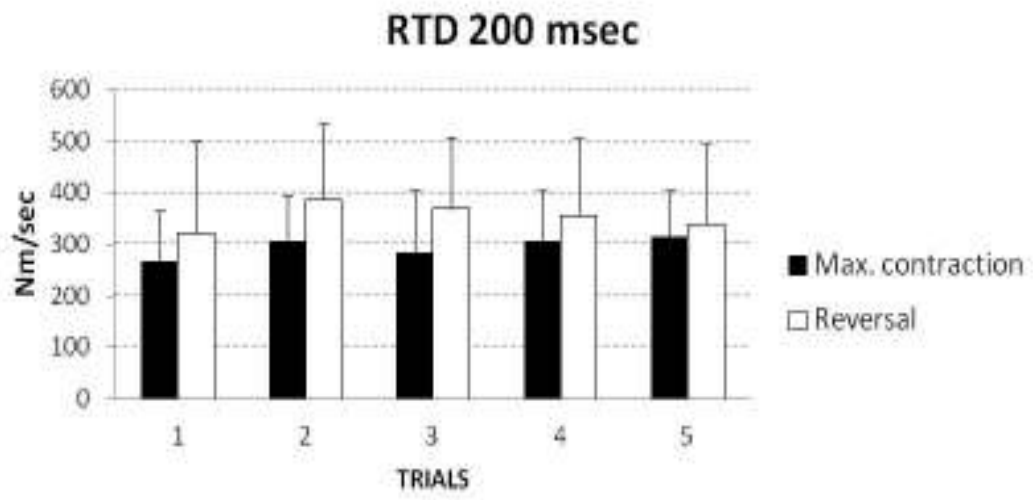

Figure XII: The rate of torque development at the first $200 \mathrm{~ms}$ at the maximal voluntary isometric contraction (black bar) and at the reversal of antagonists (white bar) 


\section{ELEFTHERIA BAKALOUDI, CHRYSTALLA PANTELI, ANTHI XENOFONTOS, ELENI BASSA, CHRISTOS KOTZAMANIDIS}

\section{Discussion}

The obtained results indicate that REV did not affect on torque output but caused an increase in RTD after $100 \mathrm{~ms}$. The effect of REV on the torque of the agonist muscle is a conflicting case because in other cases an increase of the torque output was observed (Roy et al., 1990; Kamimura et al., 2009; Kamimura et al., 2007) while in other cases not (Bohannon et al., 1986; Gabriel et al., 2001; Grabiner, 1994). A possible reason which can explain the obtained results is that the duration of the contraction of the dorsiflexors was $2 \mathrm{~s}$ and not one which according to (Kamimura et al., 2009) is the appropriate duration of the antagonist muscle. In his study (Kamimura et al., 2009) it was observed that torque of the agonist muscle increased when the preceding antagonist contraction did not exceed the $1 \mathrm{~s}$ duration. Their findings was based on the fact that interval for the antagonist potentiation by GTOs is about 1 s (Moore et al., 1991; Garceau et al., 2010). This concept explains also the obtained results considering that the dorsiflexion contraction was $2 \mathrm{~s}$.

An increase of maximum rate of torque development was found in our study, which is in agreement with the previously reported studies on this issue (Gabriel et al., 2001; Kamimura et al., 2007; Kamimura et al., 2009; Grabiner, 1994). However there is not agreement about the mechanism which caused this adaptation. Some studies found an increase of the agonist EMG (Kamimura et al., 2007; Kamimura et al., 2009; Grabiner, 1994) while in other not (Gabriel et al 2001). At this point it is very important to comment the following. The RTD after REV is affected by various factors including the neuronal one. The increase of agonist activity after REV is an indicator of neuronal involvement in RFD enhancement as indicated by relevant studies as well (Kamimura et al., 2007; Kamimura et al., 2009; Grabiner, 1994). According to some authors (Gabriel et al., 2001; Roy et al., 1990) RTD after REV may be affected by other factors such as stretch shortening cycle (SCS). This case was supported by the fact that during REV when the antagonist muscle is activated the agonist muscle is contracted eccentrically and afterwards concentrically which consequently activates RTD. However this case could be true only for dynamic actions and when the applied velocities should be appropriately high and not for any instance when the applied contraction is an isometric one.

No data exist till now which phase of RTD is been affected more after REV. According to Andersen and Aaagard (2006) the initial phase of RTD is affected by muscle contractile properties and type II muscle fibers while for intervals higher than $100 \mathrm{~ms}$ mainly by the strength increase per second. Since fiber type distribution and contractile properties were not assessed in this study these cases cannot be analyzed further in this study. The new data that emerges in this study is that the increase of RTD occurs after the first $100 \mathrm{ms,}$ while in the beginning of the contraction the RTD was lower in the agonist contraction. There is a study (L. L. Andersen, J. L. Andersen, M. K. Zebis, P. Aagaard, 2010) which can support partially the obtained finding because they reported that after a resistance training the RFD decreased in the early phase and increase in the late phase (after $100 \mathrm{~ms}$ ). However this point needs further consideration. However two cases could explain the obtained data which are related to methodological issues. Firstly we did not evaluate always the first contraction of the agonist muscle immediately after REV but the best one. Secondly we did not subtract antagonist force decay from the onset of force development of the agonist muscle (Kamimura et al., 2009).

\section{Conclusions}

In conclusion, maximum voluntary isometric contraction of antagonist muscles just before the contraction of the agonist muscles (reversal of antagonists) caused an increase of the rate of torque development of the latter indicating that REV phenomenon exist for the plantar flexors as well. Its superiority compared to maximum contraction appears after $100 \mathrm{~ms}$. Nonetheless is a new element, which needs further investigation, because it may reveal another aspect of reversal of antagonists, which may contribute to a deeper understanding of this phenomenon.

\section{References}

Aagaard P., Simonsen E.B., Andersen J.L., Magnusson P., \& Dyhre-Poulsen P. (2002). Increased rate of force development and neural drive of human skeletal muscle following resistance training. J Appl Physiol, 93(4):1318-1326.

Andersen LL, Aagaard P. (2006) Influence of maximal muscle strength and intrinsic muscle contractile properties on contractile rate of force development. Eur J Appl Physiol 96: 46-52.

Bohannon R.W. (1985). Knee extension torque during repeated knee extension-flexion reversals and separated knee extension-flexion dyads. Phys Ther 65:1052-1054.

Bohannon R.W., Gibson D.F., \& Larkin P. (1986). Effect of resisted knee flexion on knee extension torque. Phys Ther, 66:1239-1241. 
Carew T.J. (1982). Spinal cord II: Reflex action. In Kandel E.R., Schwartz J.H. eds. Principles of Neural Science. New York, Elsevier/North Holland, 293-304.

Cavagna G.A. (1977). Storage and utilization of elastic energy in skeletal muscle. Exerc Sport Sci Rev, 5:89129.

Gabriel D.A., Basford J.R., \& An, K.N. (1997). Reversal of antagonists: Effect of elbow extension strength and endurance. Arch Phys Med Rehab, 78:1191-1195.

Gabriel D.A., Basford J.R., \& An, K.N. (2001). The reversal of antagonists facilitates the peak rate of tension development. Arch Phys Med Rehab, 82:342-346.

Garceau L.R., Gray A., Fauth M.L., Hanson P., Hsu B., Yoon T., Szalkowski C., Lutsch B., \& Ebben W.P. (2010). Antagonist conditioning contractions impair agonist functioning. Proceedings of the XXVIII Congress of the International Society of Biomechanics in Sports; (R. Jensen, W. Ebben, E. Petushek, C. Richter, \& K. Roemer, editors) 199-202.

Grabiner M.D. (1994). Maximum rate of force development is increased by antagonist condition contraction. $J$ Appl Physiol, 77:807-811.

Hunt C.C. (1952). The effect of stretch receptors from muscle on the discharge of motoneurons. $J$ Physiol, 117:359-379.

Kabat H. (1950). Studies on neuromuscular dysfunction XV: The role of central facilitation in restoration of motor function in paralysis. Arch Phys Med Rehab, 33:521-533.

Kamimura T., \& Takenaka T. (2007). Potentiation of Knee Extensor Contraction by Antagonist Conditioning Contraction at Several Intensities. J Physiol Anthropol, 26(4):443-447.

Kamimura T., Yoshioka K., Ito S., \& Kusakabe T. (2009). Increased rate of force development of elbow flexors by antagonist conditioning contraction. Human Movement Science, 28:407-414.

Laporte Y., \& Lloyd D.P.C. (1952). Nature and significance of the reflex connections established by large afferent fibers of muscular origin. Am J Physiol, 169:609-621.

L. L. Andersen, J. L. Andersen, M. K. Zebis, P. Aagaard. (2010). Early and late rate of force development:

differential adaptive responses to resistance training? Scand J Med Sci Sports 20: p162-e169

Moore M.A., \& Kukulka C.G. (1991). Depression of Hoffmann reflexes following voluntary contraction and implications for proprioceptive neuromuscular facilitation. Phys Ther, 71:321-333.

Proske U., \& Morgan D.L. (1987). Tendon stiffness: methods of measurement and significance for the control of movement. A review. J Biomech, 20(1):75-82.

Roy M.-A., Sylvestre M., Katch F.I., Katch V.L., \& Lagassé P.P. (1990). Proprioceptive facilitation of muscle tension during unilateral and bilateral knee extension. Int J Sports Med, 11(4):289-292.

Sahaly R., Vandewalle H., Driss T. et al., (2001). Maximal voluntary force and rate of force development in humans - importance of instruction. European Journal of Applied Physiology, 85(3): 345-350.

Sherrington C.S. (1947). Integrative action of the nervous system. 2nd rev. New Haven (CT): Yale University Press.

Suetta C., Aagaard P., Rosted A., Jakobsen A.K., Duus B., Kjaer M., \& Magnusson S.P. (2004). Traininginduced changes in muscle CSA, muscle strength, EMG, and rate of force development in elderly subjects after long-term unilateral disuse. J Appl Physiol, 97(5):1954-1961. 